\title{
Critical Care Nurses' Knowledge and Practices Concerning Eye Care of Patients at Two Teaching University Hospitals, Egypt
}

\section{Khalil NS1*, Abd Elhameed SMI², Abdel-kader FA ${ }^{3}$, and AbdAllah}

\section{Atia $\mathbf{A}^{4}$}

${ }^{1}$ Department of Critical Care and Emergency Nursing, Faculty of Nursing, Cairo University, Egypt

${ }^{2}$ Registered nurse at Talkha central Hospital, Egypt

${ }^{3}$ Department of Critical Care and Emergency Nursing, Faculty of Nursing, Mansoura University, Egypt

${ }^{4}$ Department of Anesthesia \& Critical Care Faculty of Medicine, Mansoura University, Egypt

*Corresponding author: Nahla Khalil, Department of Critical Care and Emergency Nursing, Faculty of Nursing, Cairo University, Egypt, Tel: 1066225938; Email: nahlakhalil28@yahoo.com

\section{Abstract}

Background: Eye care is recognized as a fundamental nursing procedure essential for critically ill patients to eliminate ocular complications.

Aim of the Study: was to assess nurses' knowledge and practices concerning eye care of critically ill patients in intensive care units (ICUs).

Methods: A convenient sample of 89 nurses working in Intensive Care Units at Talkha Central Hospital and Emergency Hospital Mansoura University were included in the study. Eye care knowledge questionnaire \&nursing performance check list for eye care of critically ill patient were utilized for data collection.

Results: The majority of studied ICU nurses demonstrated correct answers regarding eye assessment, signs of eye infection, risk factors for incomplete lid closure, physical examination of eye and assessment of pupil reaction to light and Hirshberg. However, the total mean knowledge score of Talkha Central Hospital is better than Emergency hospital at Mansoura University. On the other hand, more than half of nurses in both hospitals didn't practise eye care adequately such as hand washing before applying eye care., assessing signs of infection, assessing the pupil reflex \& eye movement. Finally, no significant correlation was found between total knowledge score of the studied nurses about eye care and their performance in both hospitals ( $r=0.034$, P Value 0.751).

Conclusion and Recommendations: Nurses' practices regarding eye care of critically ill patients in (ICUs) in both hospitals were not adequate, in spite their satisfactory knowledge. Therefore, Educational initiatives should focus on weaknesses in practice noted to improve eye care of patients in ICU.

Keywords: Nurses' Knowledge; Practices; Eye Care; Critically Ill Patients; Intensive Care Units 


\section{Nursing \& Healthcare International Journal}

\section{Introduction}

Eye care is recognized as a fundamental nursing procedure essential for critically ill patients to eliminate ocular complications. But unfortunately, ICU nurse usually focuses on life-threatening problems, and anticipating and preventing eye problems are not common among the ICU team. They have an increased chance for contracting a nosocomial ocular infection from healthcare providers due to their impaired natural eye protection mechanisms which leads to decrease tear production and increase evaporation due to eye lid opening $[1,2]$.

Nurses working in ICU should have a great attention for the care to patients with critically health situations which require individual and complex interventions to meet patient's needs. In response to technological innovations in these setting critical care nurses' requiring many skills and abilities, which make constant changes in their work process. Studies show that neglection of critically ill patients eye hygiene and eye protection in (ICUs) make them predisposing for the risk of ophthalmic adverse events [3].

\section{Methods}

Descriptive exploratory research design was used in the current study. A convenient sample of 89 nurses working in Intensive Care Units at Talkha Central Hospital and Emergency Hospital Mansoura University were included in this study. The nurses were distributed as follows: (15) nurses worked at ICUs of Talkha Central Hospital and (74) nurses worked at ICUs of Emergency Hospital Mansoura University.

Two tools were utilized to collect data pertinent to this study first, eye care questionnaire it consisted of two main parts as follows: Part I: Nurses' Demographic Data which included age, gender, years of experience place of work, educational level, and previous attendance of educational program regarding eye care. Part II: Nurses' Knowledge Questionnaire Concerning Eye Care that used to assess nurses' knowledge regarding patient's eye status and eye care. The knowledge questionnaire included (18) questions in Multiple Choice Questions(MCQ) form that represents two categories: first; assessment of normal eye and pathophysiological alteration of eye status included the following (5) items; lid closure, risk factors for incomplete lid closure, lid cleanliness, corneal dryness, and signs of eye infection. Second; eye care included (5) items; eye hygiene, eye covers, eye lubricants, eye swab for culture, and antibiotics use. The Scoring system was distributed as follows: Zero $=$ no $/$ incorrect answer, $1=$ correct answer. Accordingly, those who obtained $80 \%$ or more were classified as satisfactory level, and below $80 \%$ classified as unsatisfactory knowledge level.

The Second tool was observational checklist of nurses' practices of eye care in critically ill patients that used to assess the actual nursing care provided in critical care clinical settings. It included 12 items distributed as follows: nurses' hand hygiene before and after touching the patient (3 items), physical examination of patient's eyes ( 6 items), and assessing the pertinent eye care conducted for those patients ( 3 items). Those practices items were calculated as follows: each complete done practice step $=(1)$ mark, false or missed practice step $=$ (Zero). Total scores were classified in two categories as follows: unsatisfactory practice level $(<75 \%)$ and satisfactory practice level (>75\%).

\section{Pilot Study}

A pilot study was carried out on $10 \%$ of sample size (10 nurses). To examine the feasibility and clarity of the developed tools .The necessary modifications were done. The modifications were in the form of editing of the long sentences. Cronbach's Alpha for the knowledge questionnaire was 0.732 , and test retest $(r)$ for the observational checklist was $(\mathrm{r}=0.762)$.

\section{Statistical Analysis}

After completion of data collection was tabulated and analyzed using suitable Statistical Package for Social Sciences (SPSS) version 20. The level of P value $(0.05)$ was used as the cut off value for statistical significance. Descriptive statistics was utilized in the current study such as number, percentage to describe and summarize qualitative data. Moreover arithmetic means and standard deviation (SD) was used for measuring of central tendency and dispersion respectively for normal distributed quantitative data such as knowledge and practice scores. As well, inferential statistics was utilized such as Paired sample t-test and ANOVA test for comparison of knowledge and practice scores between nurses.

\section{Results}

It is apparent from Table 1 that more than one third of nurses in Talka and Emergency Hospital Mansoura University, their age range between 25-34 years with the mean age $26.93 \pm 3.37$ \& $28.84 \pm 4.33$ respectively. Concerning nurses gender, most of nurses were female. More than one third of them in both hospitals, their years of experience ranges between 5-10 years with the mean 
5.7 and 9.9 respectively. Regarding nurses' educational level, two thirds of nurses in Talkha were bachelor graduates, while two thirds of nurses in the emergency hospital were diploma nurses graduates. Regarding the nurses area of work and the nurse patient ratio, most of nurses' in emergency hospital were working in medical ICUs and the nurse patient ratio was $1: 2$, while most of nurses in Talkha hospital were working in general ICU and the patient ratio was 1:3.

\begin{tabular}{|c|c|c|c|c|c|}
\hline \multirow{2}{*}{ Variables } & Talkha (15) & & Emergency (74) & & \multirow{2}{*}{ Significance tes } \\
\hline & $\mathrm{N}$ & $\%$ & $\mathrm{~N}$ & $\%$ & \\
\hline \multicolumn{6}{|l|}{ Age } \\
\hline $20-24$ & 3 & 20 & 16 & 21.6 & \\
\hline $25-29$ & 9 & 60 & 28 & 37.8 & \\
\hline $30-34$ & 3 & 20 & 21 & 28.4 & \\
\hline $35-39$ & 0 & 0 & 9 & 12.2 & \\
\hline Mean \pm SD & \multicolumn{2}{|c|}{$26.93 \pm 3.37$} & \multicolumn{2}{|l|}{$28.84 \pm 4.33$} & $\begin{array}{c}\mathrm{t}=1.606 \\
\mathrm{p}=0.112^{*}\end{array}$ \\
\hline \multicolumn{6}{|l|}{ Gender } \\
\hline Male & 3 & 20 & 0 & 0 & \\
\hline Female & 12 & 80 & 74 & 100 & \\
\hline \multicolumn{6}{|l|}{ Education level } \\
\hline Technical & 1 & 6.7 & 3 & 4.1 & \\
\hline Diploma & 5 & 33 & 54 & 73 & \\
\hline Bachlor & 9 & 60 & 17 & 23 & \\
\hline \multicolumn{6}{|c|}{ Years of experience } \\
\hline$<5$ years & 10 & 66.7 & 26 & 35.1 & \\
\hline 9-May & 3 & 20 & 23 & 31.1 & \\
\hline 15 -Oct & 2 & 13 & 25 & 33.8 & \\
\hline Mean \pm SD & \multicolumn{2}{|l|}{$5.6 \pm 5.7$} & \multicolumn{2}{|l|}{$9.97 \pm 6.34$} & \\
\hline \multicolumn{6}{|l|}{ Type of ICU } \\
\hline General ICU & 11 & 73.3 & 0 & 0 & \\
\hline Medical ICU & 4 & 26.7 & 74 & 100 & \\
\hline \multicolumn{6}{|c|}{ Nurse/Patient Ratio } \\
\hline $1: 3$ & 13 & 86.7 & 0 & 0 & \\
\hline $1: 2$ & 2 & 13.3 & 74 & 100 & \\
\hline
\end{tabular}

Table 1: Distribution of nurses' demographic characteristics by their area of work.

Table 2 shows that the majority of studied nurses about (91\% to $100 \%$ ) demonstrated correct answer regarding eye status that includes: assessment of eye lid \& eye lashes, signs of eye infection (91\%), risk factors for incomplete lid closure, physical examination of eye $(100 \%)$, assessment of pupil reaction to light and Hirshberg test $(98.9 \%)$. As well, the majority of nurses (94\% to $100 \%$ ) demonstrated correct answer regarding eye care except some items regarding nursing actions of patients' admission with chemical injury $(67.4 \%)$ and daily routine of eye care $(61.8 \%)$. On the other hand, approximately half of nurses (54.6\%) gave incorrect answer about frequency of lid cleaning.

It's apparent from Table 3 that half of nurses didn't practise hand washing before applying eye care. As well, all of nurses (85\% to $100 \%)$ didn't assess signs of infection and didn't assess the patient for pupil reflex \& eye movement. Regarding performing eye care of patients, all nurses' didn't maintain eye care of patient routinely, explain procedure to their patients and conduct eye care routinely and the frequency of care wasn't delineated. 


\section{Nursing \& Healthcare International Journal}

\begin{tabular}{|c|c|c|c|c|}
\hline \multirow{2}{*}{ Variables } & \multicolumn{2}{|c|}{ Incorrect answer } & \multicolumn{2}{|c|}{ Correct answer } \\
\hline & NO & $\%$ & NO & $\%$ \\
\hline \multicolumn{5}{|l|}{ Eye Status (Mean 6.20; SD \pm 0.66 ) } \\
\hline Assessment of Eyelids \& Eyelashes. & 8 & 9 & 81 & 91 \\
\hline Risk factors for incomplete lid closure. & 0 & 0 & 89 & 100 \\
\hline Frequency of lid cleanliness. & 53 & 54.6 & 36 & 40.4 \\
\hline Signs of eye infection. & 8 & 9 & 81 & 91 \\
\hline Physical examination of eye. & 0 & 0 & 89 & 100 \\
\hline Checking pupil response to light. & 1 & 1.1 & 88 & 98.9 \\
\hline Corneal Light Reflex (The Hirshberg Test) - Shining light on the eyes. & 1 & 1.1 & 88 & 98.9 \\
\hline \multicolumn{5}{|l|}{ Eye Care ( Mean 9.66; SD \pm 1.05 ) } \\
\hline Cleaning of lid. & 2 & 2.2 & 87 & 97.8 \\
\hline Nursing care of corneal dryness. & 5 & 5.6 & 84 & 94.4 \\
\hline Covering the eye. & 1 & 1.1 & 88 & 98.9 \\
\hline Frequency of changing eye covers. & 1 & 1.1 & 88 & 98.9 \\
\hline Recommendation of eye covers. & 3.3 & 3.4 & 86 & 96.6 \\
\hline Eye lubricant. & 3.3 & 3.4 & 86 & 96.6 \\
\hline Eye swab for culture. & 4 & 4.5 & 85 & 95.5 \\
\hline Antibiotics used for eye care. & 0 & 0 & 89 & 100 \\
\hline \begin{tabular}{|l|} 
Nursing care for patient admitted with chemical eye injury. \\
\end{tabular} & 29 & 32.6 & 60 & 67.4 \\
\hline $\begin{array}{l}\text { Nursing action for the patient admitted with bright red blood drainage } \\
\text { from the eye. }\end{array}$ & 17 & 19.1 & 72 & 80.9 \\
\hline \begin{tabular}{|l|} 
Daily routine of eye care. \\
\end{tabular} & 34 & 38.2 & 55 & 61.8 \\
\hline \multicolumn{5}{|l|}{ Total mean 15.84; SD \pm 1.27} \\
\hline
\end{tabular}

Table 2: Nurses' distribution of correct and incorrect answers in relation to Knowledge regarding eye care of critically ill patients $(\mathrm{n}=89)$.

\begin{tabular}{|c|c|c|c|c|}
\hline \multirow{2}{*}{ Steps } & \multicolumn{2}{|c|}{ Done } & \multicolumn{2}{|c|}{ Not Done } \\
\hline & NO. & $\%$ & NO. & $\%$ \\
\hline \multicolumn{5}{|l|}{ Hand Hygiene } \\
\hline Wash hand before procedure & 40 & 44.5 & 49 & 55.1 \\
\hline Wash hand after procedure & 87 & 97.8 & 2 & 2.2 \\
\hline Wear gloves & 89 & 100 & - & - \\
\hline \multicolumn{5}{|l|}{ Observation and Examination } \\
\hline Asses and notify signs of infection & 13 & 14.6 & 76 & 85.4 \\
\hline Inspect patient's eyes for drainage, irritation, and lesions & 11 & 12.4 & 78 & 87.6 \\
\hline Perform pupillary examination & - & - & 89 & 100 \\
\hline Assess for blink reflex & - & - & 89- & 100 \\
\hline Inspect eye movements\& noting symmetry & - & - & 89- & 100 \\
\hline \multicolumn{5}{|l|}{ Assess Eye Care } \\
\hline $\begin{array}{l}\text { Explain procedure to patient and family members if } \\
\text { present according to his conscious level }\end{array}$ & 1 & 1.1 & 88 & 98.9 \\
\hline $\begin{array}{l}\text { Apply clean moistened cloth and gently wipe each eye } \\
\text { from inner to outer canthus of eye }\end{array}$ & 79 & 88.8 & 10 & 11.2 \\
\hline Use an eye dropper or lubricant as ordered & 48 & 53.9 & 41 & 46.1 \\
\hline Maintain eye care of patient routinely & - & - & 89 & 100 \\
\hline
\end{tabular}

Table 3: Frequency distribution of nurses' done \& not done practices of eye care of critically ill patients ( $\mathrm{n}=89$ ). 


\section{Nursing \& Healthcare International Journal}

Table 4 revealed that there were no significant statistical difference in knowledge score among group of nurses, by their demographic characteristics except education $(F=5, P=0.009)$. So, the bachelor nurses demonstrated slightly higher knowledge than technical and diploma nurses .Moreover the current findings revealed no significant statistical difference in practice scores among group of nurses, by their demographic characteristics except experience where $(\mathrm{F}=3.23, \mathrm{P}=0.04)$. So, the nurses' experience below 5 years showed slightly higher practice scores, however, their practice was unsatisfactory.

\begin{tabular}{|c|c|c|c|}
\hline Demographic Characteristics & NO -89 & Knowledge Score & Performance Score \\
\hline \multicolumn{4}{|l|}{ ( } \\
\hline $20-24$ & 19 & $15.74 \pm 1.23$ & $4.21 \pm 1.03$ \\
\hline $25-29$ & 37 & $16.14 \pm 1.38$ & $4.24 \pm 1.09$ \\
\hline $30-34$ & 24 & $15.58 \pm 1.39$ & $3.83 \pm 1.09$ \\
\hline $35-39$ & 9 & $15.44 \pm 1.27$ & $4.44 \pm 0.89$ \\
\hline Significance Test & & $F=1.309, p=0.277$ & $F=1.061, p=0.370$ \\
\hline \multicolumn{4}{|l|}{ Experience } \\
\hline$<5$ years & 36 & $16.14 \pm 1.43$ & $4.44 \pm 1.16$ \\
\hline $5-<10$ & 26 & $15.57 \pm 1.06$ & $3.77 \pm 0.76$ \\
\hline $15+$ & 27 & $15.63 \pm 1.15$ & $4.11 \pm 1.08$ \\
\hline Significance Test & & $F=2.407, p=0.096$ & $F=3.233, p=0.044$ \\
\hline \multicolumn{4}{|l|}{ Education } \\
\hline Technical & 4 & $16.00 \pm 0.000$ & $4.25 \pm 1.26$ \\
\hline Diploma & 59 & $15.56 \pm 1.13$ & $4.00 \pm 0.96$ \\
\hline Bachelors & 26 & $16.46 \pm 1.45$ & $4.46 \pm 1.21$ \\
\hline Significance Test & & $F=5.009, p=0.009$ & $F=1.757, p=0.174$ \\
\hline \multicolumn{4}{|l|}{ Gender } \\
\hline Male & 3 & $16.67 \pm 0.58$ & $4.33 \pm 1.53$ \\
\hline Females & 86 & $15.81 \pm 1.28$ & $4.14 \pm 1.05$ \\
\hline $\begin{array}{ll}\text { Significance Test } \\
\end{array}$ & & $t=1.146, p=0.225$ & $t=0.309, p=0.758$ \\
\hline \multicolumn{4}{|l|}{ Nurse/Patient Ratio } \\
\hline $1: 03$ & 13 & $16.38 \pm 1.26$ & $4.31 \pm 1.03$ \\
\hline $1: 02$ & 76 & $15.75 \pm 1.25$ & $4.12 \pm 1.07$ \\
\hline Significance Test & & $t=1.683, p=0.096$ & $t=0.542, p=0.55$ \\
\hline
\end{tabular}

Table 4: Frequency distribution of knowledge and performance scores by their demographic characteristics.

It's apparent from Table 5 that there was significant statistical difference in knowledge scores between nurses, in both hospitals $(\mathrm{T}=2.6)$. As the knowledge of nurses, in Talkha Central Hospital is better than in emergency hospital Mansoura University. On the other hand, no significant statistical differences were found between nurses' regarding practices scores in both hospitals.

\begin{tabular}{|c|c|c|c|}
\hline Items & Talkha No= (15) & Emergency No= (74) & Significance test \\
\hline Knowledge score & $16.6 \pm 1.30$ & $15.69 \pm 1.22$ & $\mathrm{t}=2.617$ P 0.010 \\
\hline Performance score & $4.20 \pm 1.15$ & $4.13 \pm 1.05$ & $\mathrm{t}=0.215$ P 0.831 \\
\hline
\end{tabular}

Table 5: Comparison of knowledge and performance scores among the studied nurses by their area of work 


\section{Nursing \& Healthcare International Journal}

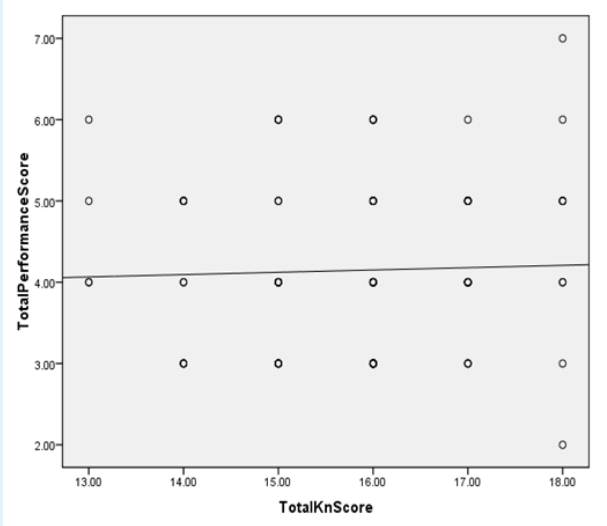

Figure 1: Correlation between total knowledge score of the studied nurses and their performance score regarding eye care

It's apparent from Figure 1 that there was no correlation between total knowledge score of the studied nurses about eye care and their performance $(r=0.034$, P0.751).

\section{Discussion}

The current study aimed to assess nurses' knowledge and practices regarding eye care of critically ill patients. Regarding nurses' knowledge concerning eye care in critically ill patients, the current study findings revealed that most of nurses had a satisfactory knowledge score regarding eye care in critically ill patients. From the researcher point of view, critical care nurses' have an adequate knowledge. This finding may have relevance to the body of knowledge that acquired among newly graduated baccalaureate nurses from their nursing curricula.

This finding is agreed with Fulbrook, et al. (2012) [4] who conducted a survey of European Intensive Care Nurses' knowledge levels about eye care and revealed that overall knowledge score of the study was satisfactory. Concerning the specific knowledge about corneal light reflex and frequency of eye care per day, the majority of studied nurses gave correct answers. This finding may have relevance to applying this procedure in clinical practice frequently. This finding is agreed with Cho, et al. (2017) [5] who studied development and validation of an eye care educational program for intensive care unit nurses' and found that most of CCN had better knowledge about corneal light reflex and frequency of eye care per day.
As well, the current study findings is partially agreed with Fashafsheh, et al. (2013) [6] who studied the impact of a designed eye care protocol on nurses' knowledge, practices about eye health status of unconscious mechanically ventilated patients at north Palestine hospitals, and found that the majority of critical care nurses' has adequate knowledge about eye swabs and eye care.

Regarding the nurses' practices regarding eye care in critically ill patients, it was found that nurses' practices scores were unsatisfactory. The researcher point of view that finding was due to considering eye care is not lifesaving procedure among the whole health team members as well as no protocol of care is followed among the nurses, in the critical care settings.

In relation to eye assessment, the majority of studied nurses, didn't assess the eye. That observation finding may have relevant to lack of training courses regarding eye care , lack of head nurses' audit and supervision and this finding is contradicted with the result of Fashafsheh, et al. (2013) [6] who studied the impact of a designed eye care protocol on nurses, knowledge, practices and on eye health status of unconscious mechanically ventilated patients at north Palestine hospitals and found that nurses' practices of eye assessment showed significant improvement after application of eye care protocol.

Moreover, our study finding revealed that most of nurses, comply with application of moistened gauze with saline and eye pads frequently. So, this finding may due to the availability of resources such saline and gauze. This finding was consistent with Kam, et al. (2013) [7] who studied eye care in the critically ill; Güler, et al. (2016) [8] who studied intensive care nurses' views and practices for eye care and both studies found that the most familiar methods for eye care is gauze socked with saline for eye hygiene.

Regarding nurses' performance of hand washing, the majority of studied nurses washed their hands after touching the patient only and wore gloves. On the other hand, more than half of studied nurses didn't wash hand before touching the patient. The researcher point of view for this observation was due to absence of written eye care protocol for nurses to follow. Moreover, the unsatisfactory level of practices of critical care nurses' may have relevance to considering the eye care is not a life threatening condition in ICU setting. This finding was also consistent with other study done by Azfar, et al. (2013) [9] who studied protocolized eye care to prevent corneal complications in mechanically ventilated patients 


\section{Nursing \& Healthcare International Journal}

in a medical intensive unit which revealed that the eye care was neglected in ICUs.

Concerning the relation between nurses' knowledge and practices regarding eye care of critically ill patients, our findings revealed no significant correlation between total knowledge score of the studied nurses about eye care and their performance. Unsatisfactory practices of nurses despite of having adequate knowledge may due to absence of nursing audit and surveillance. This result was contradicted with the study done by Fashafsheh, et al. (2013) [6] that studied the impact of a designed eye care protocol on nurses knowledge, practices and on eye health status of unconscious mechanically ventilated patients, and found that there was a relation between level of knowledge for the critical care nurses of eye care and their performance score.

The researcher was interested to investigate the differences between nurses by their demographic characteristics in relation to knowledge and practices regarding eye care in critically ill patients. However, the current finding revealed no differences in nurses' knowledge and practice except educational level in nurses' knowledge and experience in nurses' practice. The current finding is consistent with Chan, et al. (2012) [4] study results show that there were no significant relationships between the knowledge score and the sample's characteristics. As well, Similar results were seen in the study of Huang, et al. (2009) [10] who revealed no significant differences in length of nursing experience regarding knowledge. On the same line, our study findings were partially consistent with Fashafsheh, et al. (2013) [6] who revealed that educational of nearly half of the nurses were BA holders which reflects stabilized level of knowledge and practice.

\section{Conclusion}

The present study concluded that nurses' in talkha central hospital and Emergency hospital demonstrated unsatisfactory level of practice regarding eye care for critically ill patients although they have got satisfactory level of knowledge about eye care in intensive care units. Moreover, no significant correlation was found between nurses' knowledge and practice regarding eye care in critically ill patients.

\section{Recommendations}

Based on the finding of the present study, the following recommendations were made:
- Eye care should be a routine care for the critically ill patient in ICU.

- Clinical training programs about eye assessment protocol and care of critically ill patients is needed.

- Nurses frequent assessment of eye health status and care should be maintained and recorded in nursing records.

\section{References}

1. Shan H, Min D (2010) Prevention of exposure keratopathy in intensive care unit. Int J Ophthalmol 3(4): 346-348.

2. Zhou Y, Liu J, Cui Y, Zhu H, Lu Z (2014) Moisture chamber versus lubrication for corneal protection in critically ill patients: A meta-analysis. Cornea 33(11): 1179-1185.

3. Werli-Alvarenga A, Ercole FF, Botoni FA, Oliveira J, Chianca TCM (2011) Corneal injuries: incidence and risk factors in the Intensive Care Unit. Revista LatinoAmericana de Enfermagem 19(5): 1088-1095.

4. Fulbrook P, Albarran JW, Baktoft B, Sidebottom B (2012) A survey of European intensive care nurses' knowledge levels. Int J Nurs Stud 49(2): 191-200.

5. Cho OH, Yoo YS, Yun SH, Hwang KH (2017) Development and validation of an eye care educational programme for intensive care unit nurses. J ClinNurs 26(13-14): 2073-2082.

6. Fashafsheh IHD, Morsy WYM, Ismaeel MS, Alkaiasi AAE (2013) Impact of a designed eye care protocol on nurses knowledge, practices and on eye health status of unconscious mechanically ventilated patients at North Palestine Hospitals. Journal of Education and Practice 4(28): 107-120.

7. Kam KYR, Haldar S, Papamichael E, Pearce KCS, Hayes M, et al. (2013) Eye care in the critically ill: A national survey and protocol. Journal of Intensive Care Society 14(2): 150-154.

8. Güler EK, Eser I, Fashafsheh IH (2016) Intensive Care Nurses' Views and Practices for Eye Care: An International Comparison. Clinical Nursing Research 26(4): 504-524.

9. Azfar MF, Khan MF, Alzeer AH (2013) Protocolized eye care prevents corneal complications in ventilated patients in a medical intensive unit. Saudi J Anaesth 7(1): 33-36. 


\section{Nursing \& Healthcare International Journal}

10. Huang H, Chuang Y, Chiang K (2009) Nurses' physical restraint knowledge, attitudes, and practices: The effectiveness of an in-service education program. J Nurs Res 17(4): 241-248. 